\title{
Les distributions de prix au temps de Jules Ferry: deux exemples tarnais
}

\section{Nicole Le-Pottier-Prévost}

\section{(2) OpenEdition}

\section{$\checkmark$ Journals}

Édition électronique

URL : http://journals.openedition.org/trema/1852

DOI : $10.4000 /$ trema.1852

ISSN : 2107-0997

\section{Éditeur}

Faculté d'Éducation de l'université de Montpellier

\section{Édition imprimée}

Date de publication : 1 décembre 1997

Pagination : $35-50$

ISSN : 1167-315X

\section{Référence électronique}

Nicole Le-Pottier-Prévost, "Les distributions de prix au temps de Jules Ferry: deux exemples tarnais », Tréma [En ligne], 12-13 | 1997, mis en ligne le 01 décembre 1997, consulté le 14 novembre 2019. URL: http://journals.openedition.org/trema/1852 ; DOI : 10.4000/trema.1852

Ce document a été généré automatiquement le 14 novembre 2019.

Trema 


\title{
Les distributions de prix au temps de Jules Ferry : deux exemples tarnais
}

\author{
Nicole Le-Pottier-Prévost
}

\section{Ouverture}

\section{Lavaur1, 1884 - Extrait du Journal de Lavaur, 24 août 1884.}

1 « Distribution des prix à l'école laïque.

«Dimanche dernier, l'Ecole laïque des filles, de Lavaur, célébrait sa fête de famille: la distribution des prix, sous la présidence de M. Compayré, notre vaillant député.

«Près de mille personnes, appartenant à toutes les classes de la Société, se pressaient dans la halle élégamment décorée. La plupart des membres du Conseil municipal, presque tous les fonctionnaires accompagnés de leurs dames, faisaient cortège à M. le Président. Les exercices de ces jeunes élèves ont été charmants: des chants, des chœurs où dominait la note patriotique, en composaient le programme. Les plus petites filles comme les plus grandes ont obtenu un franc et légitime succès.

"Les applaudissements du public s'adressaient autant aux élèves qu'à leurs habiles maîtresses. Le Député, dans un discours improvisé et fortement applaudi, a raconté l'histoire de cette école, créé de toutes pièces par la population républicaine de Lavaur, si florissante aujourd'hui en dépit des sinistres prophéties des adversaires de l'instruction et de l'opposition que le conseil municipal d'antan fit à sa venue au monde.

«En passant, il fait l'éloge de l'«association du Sou des Écoles lä̈ques » qui, avec ses modestes ressources, est parvenu à assurer la gratuité absolue des fournitures classiques. Il explique ensuite ce qu'on doit entendre par École laïque dont les ennemis de nos institutions ont fait un monstre affreux. Il termine enfin en donnant quelques conseils à ces jeunes enfants qui doivent être un jour, selon un mot de M. de Rémusat, épouses et mères de citoyens.

"Nous allions oublier le coup de griffe fort galamment décoché contre le R. P. Escande. C'est tout ce que méritait la plate diatribe insérée dans le Nouvelliste du 17 août. 
"Seize prix d'honneur avaient été offerts à M ${ }^{\text {lle }}$ Simon, par M. le Ministre de l'instruction publique, M. le Député, M. le Sous-Préfet, la Municipalité, l'« association du Sou des Écoles ", M. Gontier et des anonymes. Ils ont été distribués aux élèves les plus méritantes.

"Ces prix d'honneur et la nombreuse assistance qui se pressait dans la halle sont la preuve des sympathies et de la bienveillance de la population vauréenne pour notre École laïque et pour $M^{\text {lle }}$ Simon. Elle peut être fière de son cuvre. Que l'intelligente directrice nous permette de lui adresser les remerciements les plus sincères des enfants et de leurs parents. ॥

\section{Castelnau-de-Montmiral'2, 1886 - Extrait du Bon citoyen des campagnes : organe républicain des populations rurales du Tarn, 22 août 1886}

\section{2 «Distribution des prix aux élèves des écoles communales}

"La distribution des prix aux élèves des écoles communales, qui a eu lieu dimanche dernier, était présidée par notre sympathique député, M. Jaurès, ayant, à sa droite, M. Tornier maire et conseiller général ${ }^{3}$, à sa gauche M. Montjoie, inspecteur primaire, qui tient à coeur tous les ans de venir assister à cette joyeuse fête de l'enfance. Sur une estrade artistement ornée, on remarquait les conseillers municipaux, les principales notabilités de la ville, enfin bon nombre d'instituteurs qui, eux aussi, voulaient participer à cette réjouissance. M. Ressiguier, instituteur, chargé de prononcer le discours d'usage a choisi pour sujet, l'Histoire. Nous félicitons de tout cœur ce zélé instituteur qui, dans une dernière leçon, a su si bien faire comprendre aux enfants quels seraient les grands avantages qu'ils pourraient retirer de l'étude de l'histoire.

"Son discours a été entrecoupé par de nombreux applaudissements. M. Jaurès prend aussitôt la parole. Il remercie M. le maire de l'insigne honneur qu'il lui a fait en lui accordant le fauteuil de la présidence. Il est tout heureux, a-t-il dit, de se retrouver au milieu des enfants qu'il aime tant et pour lesquels il se promet de faire tant de bien. Puis, s'adressant particulièrement aux garçons, il les a engagés, dans un discours très éloquent, à suivre les excellents conseils que vient de leur donner leur maître; il leur a dit que la France, cette France qui est chère à tout homme de cour, ne comptait que sur la jeunesse pour déchirer ce voile noir tendu depuis seize longues années sur deux provinces de l'Est. Son discours a été couvert d'applaudissements enthousiastes. "On a ensuite procédé à la lecture du palmarès. Il s'est produit un petit incident que l'on ne saurait passer sous silence. Une gentille petite demoiselle, B. G., après avoir prononcé d'aimables paroles, a offert un magnifique bouquet à M. Jaurès, puis, se retournant vers ses camarades, a crié: Vive la République! Vive M. Jaurès! et s'est retirée en toute hâte. M. le président s'est empressé de descendre de l'estrade pour aller embrasser cette charmante fillette. Un second bouquet a été offert à $M$. le maire par une autre élève.

Nos plus sincères félicitations aux maîtres et aux maitresses qui ont organisé une si imposante cérémonie. Nous en conserverons un excellent souvenir."

L'origine de cette cérémonie scolaire est lointaine. Un Jésuite portugais l' «inventa ", puisque ce fut à Coïmbra en 1558 que, pour la première fois, on releva »le plaisir délicat que procuraient ces dons, de l'éclat glorieux d'une remise publique "4. Le rituel est déjà en place, puisque la cérémonie comporte une comédie, des discours et des épigrammes, des chants et la distribution des prix par un élève. C'était le couronnement public d'une pédagogie fondée sur le recours constant à l'émulation dans un enseignement qui a retiré l'enfant du monde pour l'éduquer. "De toutes pièces, les éducateurs vont organiser motivations et joies purement scolaires: ce sera le monde de l'émulation. $\|^{5}$ 
Les comptes rendus par la presse locale des distributions des prix à Lavaur et à Castelnau-de-Montmiral dénotent un renversement de perspective. Dans les années 1880 la finalité de cette célébration publique n'est plus purement pédagogique. Les représentants du pouvoir ont ravi le premier rôle aux élèves et à leurs maîtres.

\section{Un instrument politique}

4 Le cérémonial décrit dans les deux textes est le même, mais la tonalité diffère : tandis qu'à Lavaur, unvaillant député commémore une victoire toute récente dans la guerre scolaire, à Castelnau-de-Montmiral son sympathique collègue célèbre le consensus républicain. Le contraste met en lumière la fonction la plus immédiate de la cérémonie des prix dans ces années qui est de contribuer au succès du projet républicain sur son terrain de prédilection, l'école.

\section{"Près de mille personnes se pressaient dans la halle... »}

5 Dans les articles on s'attache à souligner l'importance de l'assistance, le nombre et la qualité des notables qui siègent à la tribune à côté du président de la cérémonie. Ce n'est pas un simple poncif journalistique. Les distributions des prix sont l'occasion pour les amis de la République de se compter. C'est ainsi que, lorsque Gabriel Compayré, député de fraîche date, préside sa première distribution des prix à l'école communale de garçons, le Journal de Lavaur note: "Comme tous les ans, un public nombreux et choisi avait répondu à l'appel de l'honorable directeur; seules certaines personnes considérables de Lavaur qui assistaient tous les ans à cette fête de famille, ont cru devoir s'abstenir cette fois... $1{ }^{6}$

6 La bataille politique autour des lois scolaires de 1882 à 1886 prend souvent la forme d'un débauchage des élèves de l'école adverse et confère aux familles un rôle d'arbitre ; leur présence est donc très attendue dans les distributions de prix. A l'importance de la foule on mesure le succès de l'école.

$7 \quad$ Les campagnes orchestrées à plusieurs reprises par l'Église contre l'école sans Dieu ont utilisé les familles comme masse de manœuvre. L'attaque d'ensemble menée par le clergé catholique contre les manuels de morale et d'instruction civique «neutres », qui furent substitués aux catéchismes à la suite de la loi de 1882, a largement usé de deux armes détenues par les "pères de famille »: la pétition et, plus grave, le retrait des enfants de l'école publique. Or, le département du Tarn s'est particulièrement illustré dans cette première guerre des manuels ${ }^{7}$; il fut le lieu des premiers incidents et fournit à lui seul le cinquième des prêtres sanctionnés. Et dans le Tarn, le Vaurais, région très attachée au catholicisme, fut en première ligne. D'abord l'auteur d'un des manuels incriminés n'était autre que Gabriel Compayré ${ }^{8}$; ensuite, l'un des premiers sanctionnés fut Étienne Mazas, maire de Lavaur, qui avait entraîné la commission scolaire de son arrondissement à soutenir la révolte des pères de famille : il fut révoqué par le préfet, Léon Bourgeois, futur ministre de l'Instruction publique9. En 1884, le souvenir de ces événements, vieux de moins de deux ans, contribue sans nul doute au climat de paix armée qui entoure la distribution des prix de l'école laïque de filles. 


\section{La représentation du pouvoir républicain}

"L'insigne honneur de la présidence »... Le président de la cérémonie est le personnage clé. Représentant du pouvoir républicain désigné par le préfet, il est investi du contrôle de la parole : il est le principal orateur et les éventuels autres discours sont placés sous sa responsabilité. A l'époque, la législation en vigueur qui date de l'Ordre moral (arrêté du 29 octobre 1873, art. 4) stipule qu'« Aucun discours ne devra être prononcé, dans les fêtes scolaires dont il est parlé aux articles précédents, s'il n'a reçu au préalable, l'approbation du président ». Il s'agissait alors d'empêcher les républicains de profiter de cette tribune pour développer leurs idées. La situation s'est inversée, la prudence reste cependant de mise.

9 Pour exercer cette fonction, on choisit des élus républicains, députés ou maires, chaque fois que cela est possible. Les fonctionnaires, préfets, sous-préfets ou inspecteurs primaires, prennent le relais lorsque les élus risquent de "manquer de prudence", selon l'expression pudique employée par les inspecteurs primaires pour désigner les opposants indésirables ${ }^{10}$. Cependant la solution administrative n'est qu'un palliatif; rien ne vaut un élu du peuple pour représenter la République éducatrice. Ainsi en 1881, dès que fut connue la victoire d'un républicain sur un candidat bonapartiste dans le Vaurais, le sous-préfet s'empressa de céder la présidence de la distribution des prix au nouveau député. A Castelnau-de-Montmiral, Jaurès étrenne, ou peu s'en faut, son premier mandat national : il a été élu aux législatives d'octobre 1885 sur une liste d'union républicaine à la suite d'une campagne autour du programme scolaire de Ferry. Il avait alors vingt-six ans...11

"Dans un discours improvisé et fortement applaudi,.." Placé sous la responsabilité du président, et la plupart du temps prononcé par lui, le discours de distribution des prix a une fonction très immédiatement politique. Alors que dans les lycées, le discours, exercice littéraire confié à un professeur, exprime moins directement des préoccupations politiques ou sociales ${ }^{12}$, à l'école primaire, l'orateur principal, qui représente la République, utilise cette tribune pour répéter les vérités simples sur lesquelles repose la légitimité de l'enseignement laïque. Il s'agit de montrer aux enfants et aux parents l'enjeu de l'instruction, de justifier l'enseignement primaire et ses orientations, au regard de leur utilité sociale. Il s'agit enfin de redire les grands principes moraux et civiques que l'école est chargée d'inculquer. Pour les «grands du certificat d'études ", c'est aussi la dernière leçon que l'école leur administre avant de les lâcher, non sans inquiétudes, dans la vie. Aussi les discours ont-ils un contenu téléologique et moralisant et les allusions à l'actualité y sont fréquentes.

11 Les discours de Jaurès et de Compayré, tels qu'ils transparaissent dans les comptes rendus, n'échappent pas aux lois du genre. Jaurès a déjà une solide réputation d'orateur. La quiétude de la situation locale lui permet de prolonger le thème du discours de l'instituteur, l'Histoire, par des considérations sur le rôle prépondérant de la jeunesse dans le grand dessein national : l'effacement de la défaite de 1870 et le recouvrement des provinces perdues. Gabriel Compayré, lui, prononce un discours d'intérêt plus local. On étrenne ici l'école laïque de filles dont l'histoire est exemplaire. Elle fut imposée à un maire réactionnaire par les républicains locaux et son succès, obtenu dans la tourmente de la guerre des manuels de morale, est pour eux un sujet de fierté qui justifie l'ampleur donnée à la distribution des prix de 1884 et la place centrale de ce thème dans le discours du député ${ }^{13}$. Comme un général au lendemain d'une 
victoire, Compayré distribue les témoignages de satisfaction à la population républicaine, au Sou des écoles, à la directrice enfin dont la personnalité a beaucoup compté dans la réussite de la greffe laïque. Ce discours est aussi celui d'un chef de parti, qui ne résiste pas à la tentation de polémiquer avec l'organe orléaniste local ${ }^{14}$. "Sur une estrade artistement ornée... » Les divers lieux possibles pour la distribution des prix se résument à deux catégories. Ou le pouvoir républicain est reçu à l'école, comme à Castelnau-de-Montmiral, et la fête garde le caractère familial et scolaire généralement souhaité par les instituteurs, ou la cérémonie est "portée sur la place publique » (la halle, le théâtre ou tout autre vaste lieu de réunion), comme à Lavaur, ce qui en fait une démonstration de force républicaine, au même titre que le 14 juillet. La taille de la commune n'est pas déterminante dans ce choix. A l'intérieur ou hors de l'école, les éléments essentiels du décor sont les mêmes: l'estrade pour les invités d'honneur, les drapeaux, la table chargée de livres et en bas une assistance encore séparée en deux groupes, les enfants avec leurs maîtres d'un côté et de l'autre les familles et le public.

\section{Une pédagogie par le don}

13 Le cérémonial de la distribution des prix s'organise autour du geste de donner. La République laïque prolonge par une distribution de livres un don fondamental : celui de l'instruction. Cet acte contient plusieurs leçons, il met en scène le rôle de l'instruction élémentaire dans la société républicaine et la place du livre dans l'instruction élémentaire.

\section{Ceux qui donnent}

14 L'Etat républicain récompense les meilleurs écoliers. En réalité, les donateurs sont divers et leur diversité même permet de saisir l'imbrication des volontés qui ont abouti aux lois scolaires.

Les distributions de prix constituent un des meilleurs moyens d'intervention privée en faveur de l'instruction; la Ligue de l'enseignement l'a donc employé avant 1879 pour propager les idées républicaines dans l'école ${ }^{15}$. Dans ce but, elle a dressé des listes de livres à offrir en prix et s'est même faite libraire afin de procurer à ses adhérents des livres à meilleur marché. Puis la victoire du parti républicain infléchit les objectifs : il convient maintenant de consolider l'action du gouvernement et non plus de la combattre. L'activité de la Ligue se concentre sur l'éducation patriotique à partir de 1881 et elle contribue largement à la coloration civique donnée aux distributions de prix laïques. Après 1900, tout en continuant son office de conseiller et de fournisseur auprès des donateurs - associations laïques, communes ou Caisses des écoles - elle change de terrain d'action et s'occupe surtout des œuvres post-scolaires ${ }^{16}$. Dans le Tarn, le Sou des écoles est alors plus actif que la Ligue : il aide les familles nécessiteuses à envoyer leurs enfants à l'école et, on le voit ici, offre des prix aux distributions.

16 Cependant, dans certains cas bien délimités, comme à Lavaur en 1884, l'Etat participe directement au don. L'étude des dossiers des concessions de prix par le ministère de l'instruction publique ${ }^{17}$ montre les conditions de cette intervention. Les motivations, dans la très grande majorité des cas, sont d'ordre politique, qu'il s'agisse d'aider un candidat républicain à la veille d'une élection disputée, de soutenir l'action des écoles 
laïques contre leurs rivales congréganistes, de témoigner de l'influence du parti républicain et de la solidarité du pouvoir avec ses soutiens locaux. Beaucoup plus rarement, le geste ministériel sanctionne un taux exceptionnel de réussite au certificat d'études. Instrument politique, la concession ministérielle va là où elle est la plus efficace : on remarque qu'elle privilégie les chefs lieux de canton, les bourgs ruraux ou les petites villes d'une certaine importance électorale. Elle vise aussi la plus grande efficacité pédagogique : le livre offert par le Ministre est destiné au meilleur élève de la Classe de fin d'études. Il accompagne un moment crucial, le passage de l'école à la vie active.

17 Les libéralités gouvernementales gardent toujours un caractère exceptionnel et incitatif. L'effort principal incombe aux communes, directement ou par le biais de subventions aux caisses des écoles. Les apports de l'Etat ou des donateurs privés viennent encourager la générosité municipale ou la suppléer dans le cas de communes hostiles, indifférentes ou indigentes.

\section{Ceux qui sont récompensés}

18 On donne les livres aux élèves mais les motivations des donateurs révèlent plusieurs niveaux dans les destinataires symboliques de la récompense: la commune républicaine qui fait des efforts pour son école, la population qui veille à l'assiduité de ses enfants, les maîtres qui obtiennent de bons résultats.

La distribution des prix est la matérialisation du classement, de la chaîne de sanction bons points, croix d'honneur, etc. - qui, toute l'année, assigne une place à chaque élève et clôt chaque exercice. Ce classement est donné comme une valeur absolue, contenant en elle-même sa propre fin. Il ne se justifie pas par une utilité pédagogique. Contrairement à ce qui se passait dans l'enseignement mutuel, il ne sert pas à moduler la progression de l'enfant dans les différentes matières. Les palmarès distinguent les différentes matières enseignées, mais l'idéal à atteindre reste le prix d'honneur, tout au sommet de la hiérarchie, qui consacre une supériorité encyclopédique, et par là même un peu abstraite, couronnant un stéréotype : le bon élève. Celui-ci est défini avant tout par les buts qui ont été fixés par l'enseignement. A la fois instruction et éducation, l'enseignement primaire réunit dans une conception un peu manichéenne les connaissances acquises et le comportement, les aptitudes morales et intellectuelles: "Les républicains donnent à l'école comme objectif l'adulte positif et non plus l'adulte croyant, ils ne lui donnent pas l'enfant $»^{18}$.

\section{Hoche ou Charlemagne?}

On l'a vu, la distribution des prix met en contact l'école, maîtres et élèves, avec une société matérialisée par une assistance hiérarchisée : sur l'estrade, les officiels, bardés de leurs décorations et munis de la parole, plus bas, le peuple des parents, dont la présence et le nombre sont le moyen d'expression, comme un vote en faveur de l'école laïque. Elle donne ainsi lieu à deux « lectures » du rôle social de l'instruction primaire qui parfois coexistent et parfois s'opposent.

21 La première fait de la distribution des prix l'image scolaire de la récompense des talents telle que la pratiquerait la société républicaine. 
"Avez-vous réfléchi, mes enfants, à ce que signifient ces mots : la distribution des prix? Ils signifient: un jour où chacun est récompensé selon ses mérites, où chacun obtient le rang dont il est digne, la part d'honneur qu'il a gagnée par son travail, par sa conduite et par ses capacités.

"Cette même justice, vous la retrouverez dans le courant de votre vie, elle ne se démentira pas. Ne croyez pas ces esprits chagrins qui vous diront que dans le monde l'injustice domine. Il n'en est rien; dans une démocratie, ce sont les plus capables et les plus dignes qui ont le plus de chances de s'élever aux postes les plus hauts. ", déclare-t-on aux écoliers de la rue de Pontoise en $1887^{19}$.

\section{celle qui privilégie l'image de l'enfant pauvre, méritant et appliqué, triomphant à} l'école de l'enfant riche mais paresseux.

"De même, c'est la foi dans les bienfaits de l'éducation qui tend à escamoter les conflits de classe au sein de la société républicaine. L'école laïque, en effet, fait, au moins en droit, voisiner sur les mêmes bancs les enfants de toute origine, riches et pauvres, ouvriers et bourgeois. Le classement scolaire a la singulière vertu de redresser les hiérarchies : l'enfant mal vêtu qui triomphe le jour de la distribution des prix, image usée et touchante de la mythologie laïque, est vraiment au cœur de cette sensibilité. C'est que le seul malheur insurmontable au pauvre, c'est l'ignorance: ou plutôt c était, puisque la République l'a vaincue; et c'est aussi que les maîtres de la Troisième République croient à la contagion de leur classement: en généralisant l'instruction, on l'imposera à la place des autres hiérarchies, et on viendra à bout des discriminations sociales ${ }^{20}$.

Cette conception fait bien de l'école un autre monde qui, dans une vision utopique de l'instruction, sera le moteur du progrès social. L'école et ses maîtres espèrent exporter leurs valeurs mythologiques - la prépondérance du travail sur la fortune, la solidarité, l'égalité des conditions devant le savoir - dans la société pour la régénérer. De ce courant témoigne Véritéd'ÉmileZola et son leitmotiv : «l'école de demain vaudra ce que 
vaudra l'instituteur. C'est l'instituteur laïque, l'instrument de vérité et de justice, qui seul peut sauver la Nation, lui rendre son rang et son action dans le monde... " ${ }^{21}$.

Ces deux images se rejoignent toutefois dans l'espérance qu'elles ont engendrée d'une promotion sociale par l'instruction. L'enseignement primaire, dont on a dit qu'il ne formait qu'à un seul métier, celui d'instituteur, a tout de même permis l'accession d'élèves des milieux populaires à des emplois plus sûrs et mieux rémunérés, en même temps que mieux considérés que ceux de leurs parents. Aux bons élèves quelque espérance était donnée de trouver un emploi dans les « écritures » après le certificat d'études.

\section{Ce qu'on donne}

En donnant des livres, on espère prolonger hors de l'école (pendant les vacances ou dans la vie) les effets de l'instruction élémentaire, dont la lecture est l'instrument essentiel.Divers éléments - le format, la toile rouge des couvertures, les dorures, l'illustration - concourent à faire du livre de prix un monument destiné à impressionner durablement les enfants et leur famille. Les circonstances de l'entrée de ces livres dans des foyers dont ils constituent bien souvent l'unique bibliothèque, renforcent leur efficacité pédagogique.

L'analyse du contenu des livres offerts comme prix d'honneur donne des indications sur les leçons que l'école primaire a voulu transmettre ainsi que sur la fonction qu'elle a assigné à la lecture ${ }^{22}$. On peut esquisser le profil du lecteur adulte ainsi formé : un éternel écolier qui agrandit son musée personnel des connaissances utiles, sous la protection tutélaire du travail, de la patrie et de la République.

\section{Les leçons d'un débat}

Vint l'heure des remises en cause. En 1905, la presse se fait l'écho de l'opinion de certaines municipalités de très grandes villes (Paris et Lyon) qui préféreraient employer les sommes qu'elles consacrent à l'achat de livres de prix à l'organisation de colonies scolaires, dont l'usage s'étend alors. La nouvelle sème l'effroi chez les fournisseurs, éditeurs et libraires, et suscite dans les milieux pédagogiques un débat sur l'opportunité de telles cérémonies, dont on trouve l'expression dans la plupart des revues consacrées à l'enseignement primaire en 1906 et 1907. Les arguments échangés révèlent les enjeux pédagogiques, politiques et sociaux des distributions de prix.

\section{Dissonances}

Dans les années 1900-1910 s'esquisse une série de bilans qui donnent de l'instruction populaire une vision assez pessimiste. L'affaire Dreyfus a montré que les «lumières » n'étaient pas aussi répandues que le promettait l'enseignement pour tous. Dans Vérité ,Zola illustre l'opinion de ceux qui voient dans l'Affaire un échec, momentané certes, des instituteurs. La vérité est bien longue à triompher. Les tentatives pour développer l'instruction populaire en dehors de l'école, à travers bibliothèques et universités populaires, ne sont pas de francs succès. Mais c'est surtout sur le terrain social que les espoirs placés par la République opportuniste dans le développement de l'instruction élémentaire reçoivent de cruels démentis. La radicalisation des conflits du travail et le 
renforcement des courants socialistes après 1900 ont entamé le mythe de l'unanimité nationale entretenu par l'école. L'instruction du peuple ne signifie pas la fin des antagonismes sociaux, le bruit et la fureur qui s'élèvent des querelles entre l'école de Dieu et l'école sans Dieu ne suffisent pas à masquer les revendications sociales. Le syndicalisme gagne même le monde des instituteurs et des discordances se font entendre dans les rangs des « hussards noirs".

On se demande, en 1905, s'il faut brûler les livres de prix, sur un ton plutôt feutré. La question n'est pas primordiale, on s'en doute, mais on discerne, en amont des positions qui s'expriment alors, le poids d'un problème plus vaste, celui du rôle de l'école. Ce n'est plus seulement l'égalité politique qui est en cause, mais l'égalité sociale.

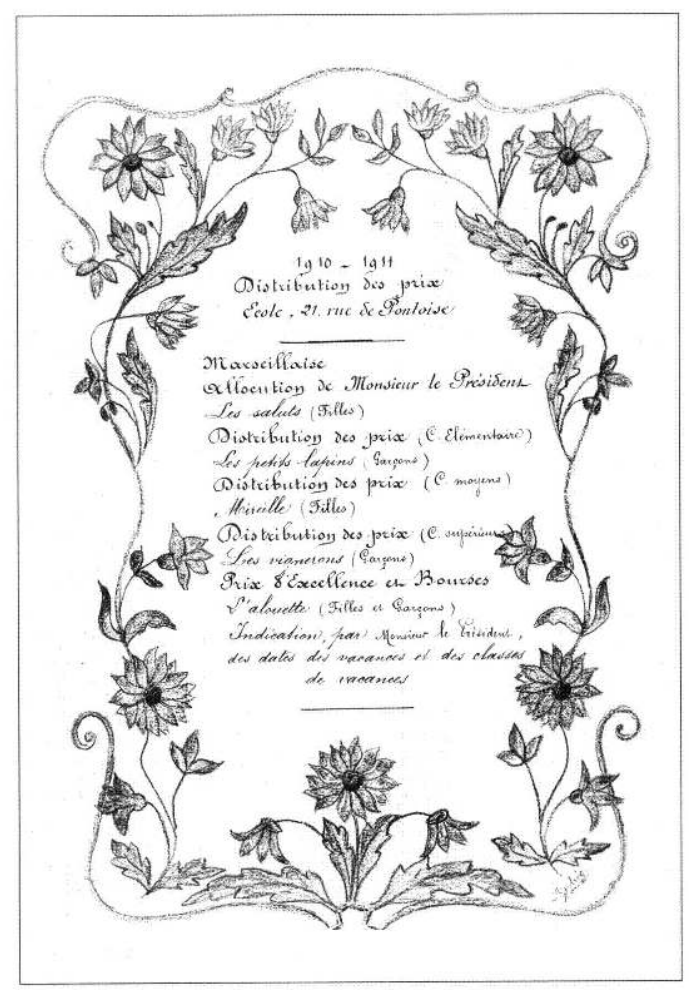

\section{Les contradictions pédagogiques}

Les pédagogues s'accordent en général pour considérer que les récompenses ne sont que des pis-aller sur le plan éducatif. Leurs réticences sont d'ordre moral d'abord, et Pauline Kergomard est sans doute celle qui a le mieux exprimé cet aspect : « En bonne et saine morale, en morale pure, la récompense consiste dans le sentiment de bien-être intérieur qu'éprouve celui qui a fait pour le mieux. Ajouter à ce sentiment l'appât d'un objet matériel, c'est le rabaisser. Aussi suis-je personnellement hostile aux récompenses matérielles ", 23écrit-t-elle.

Ceux qui défendent les récompenses, et les punitions, les dépeignent comme un mal nécessaire, parce que le sentiment du devoir est un idéal bien trop élevé pour des consciences enfantines, chez des êtres "qui n'ont pas encore la liberté ni la raison "24. Encore faut-il user de ce ressort psychologique avec modération.

34 L'argument est retourné par le camp adverse qui déclare les récompenses " immorales » car "récompenser c'est paraître supposer que l'état normal est de faire le mal " 25. La critique pédagogique s'attache également à l'objet de la récompense: 
matérialisation du classement, le prix sanctionne le résultat et non la progression, elle distingue l'élève «bien doué » et pas toujours le plus méritant. Aussi, écrit Ferdinand Buisson, "il faudrait de plus trouver une manière d'encourager et de louer l'effort fait même par un élève d'intelligence médiocre pour gagner quelques points ${ }^{26}$.

\section{Les contradictions sociales}

Les sentiments sur lesquels joue l'émulation - orgueil, esprit de compétition et individualisme - sont en contradiction avec l'éducation fondée sur la solidarité que souhaite mettre en œuvre une frange importante des instituteurs. C'est ce qui s'exprime dans les vœux sur l'éducation sociale à l'école primaire par le Deuxième Congrès des Amicales d'instituteurs et d'institutrices publics de France (Bordeaux, 1901).

«L'éducation sociale n'entraîne point la création d'un enseignement nouveau, elle consistera dans le meilleur parti à tirer, au profit de l'idée de solidarité, du mode de groupement des élèves, du système disciplinaire, des nécessités et des faits journaliers de la vie scolaire, des enseignements des œuvres de mutualité et des institutions post-scolaires, [...] Le Congrès est d'avis que dans un État démocratique toutes les distinctions honorifiques doivent être supprimées. »

Cette motion fait passer l'éducation sociale par la « pleine possession [pour les instituteurs] de toutes les prérogatives des citoyens libres", par le refus du "chauvinisme belliqueux" dans l'enseignement de l'histoire et la revendication de l'école unique.

Dans les revues pédagogiques d'inspiration socialiste, s'ajoute une remise en cause de la signification sociale que la République opportuniste et, après elle, la République radicale, ont voulu donner aux distributions de prix : celles-ci proposent une image déformée de la réalité des rapports sociaux. "Récompenser les actions de l'enfant, c'est encore très mal le préparer à la vie, qui ne récompense point comme on le fait à l'école ${ }^{27}$.

La dénonciation du fossé qui sépare les deux écoles, celle des privilégiés et celle du peuple, est toujours présente, explicite ou implicite, dans les argumentations développées à propos des prix par les groupes d'opinion qui ne se reconnaissent pas dans l'idéologie scolaire officielle ${ }^{28}$. Le souci de ne pas accentuer les différences plaide en faveur du maintien des distributions de prix. : "On ne chicane pas, ce me semble, les récompenses aux élèves de l'enseignement secondaire. On se demande s'il n'est pas injuste de priver les enfants du peuple de récompenses, alors que les fils de privilégiés de la fortune en ont " 29.

\section{Des distributions des prix quand même}

Les instituteurs syndicalistes sont les seuls à demander la suppression définitive des distributions de prix. Les autres mouvements d'opinion concluent en faveur du maintien de ces cérémonies sous une forme légèrement amendée (Manuel général de l'instruction primaire), libérées de la présence des personnalités politiques (Le Volume) ou profondément transformées (Revue de l'enseignement primaire), parce que "même avec l'organisation actuelle, si défectueuse qu'elle soit, les enfants, les parents et les maîtres sont solidaires. Il y a quelque chose qui plane au-dessus de ces cérémonies, qui inspire parfois plus d'un discours heureux, c'est l'idée du pays, c'est la notion de la société moderne, c'est le sentiment d'affection qui anime tous vis-à-vis de la nation républicaine. Il pénètre toujours un peu de l'âme de la France dans le cœur des assistants, un jour de distribution des prix ${ }^{30}$. 


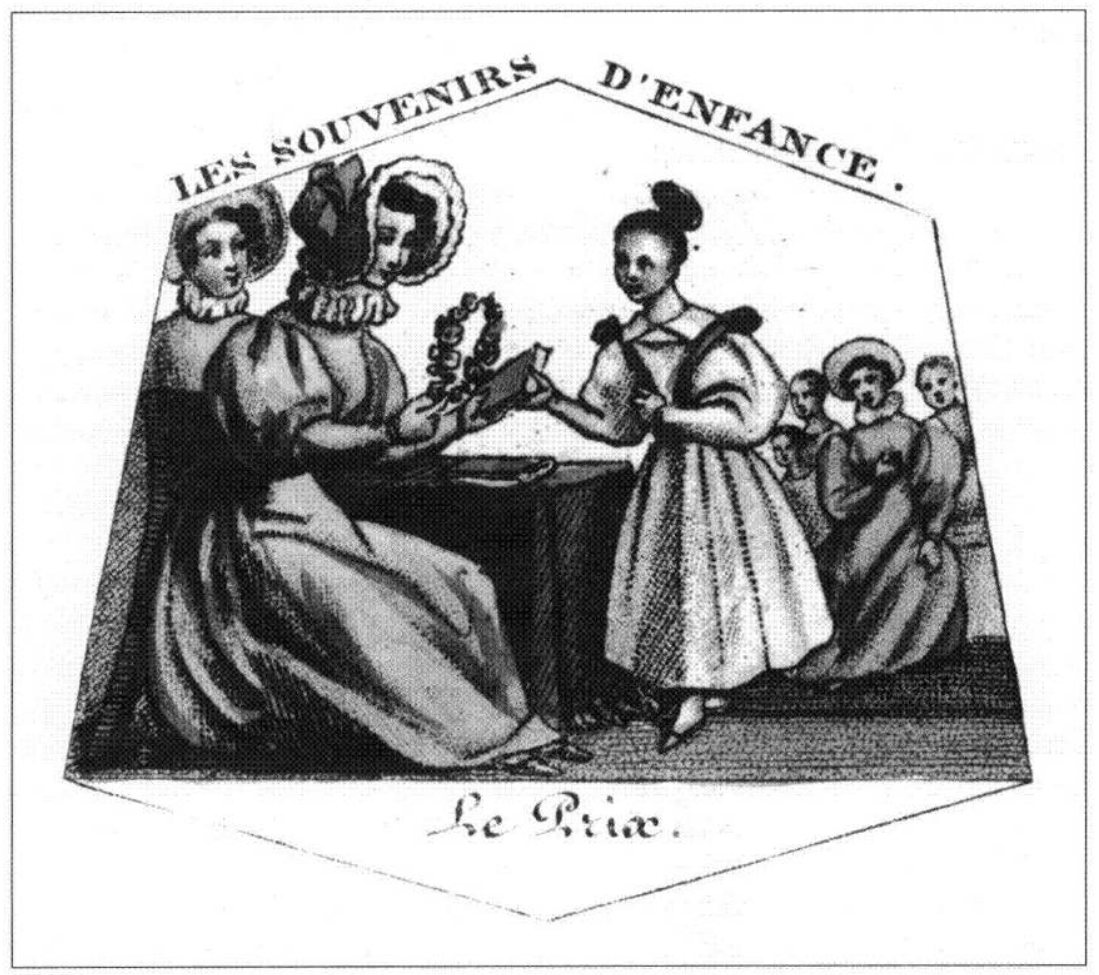
retrouve les accents des promoteurs des Fêtes d'enfants. Né au sein de la Ligue de l'enseignement en 1881, ce mouvement militait pour l'organisation des grandes fêtes conviant tous les enfants d'une ville à sortir de leurs écoles pour défiler en chantant des hymnes patriotiques. Comme les défilés du 14 juillet dont elles étaient la réplique enfantine, ces fêtes se rattachaient explicitement à la tradition de 1790 telle que Michelet l'avait transmise. La Ligue a assez vite abandonné la promotion de ce type de manifestations dont le caractère trop «militaire» soulevait des objections dans ses propres rangs, surtout après l'alerte boulangiste, mais cet épisode a attiré l'attention sur la nécessité de proposer une alternative laïque aux fêtes religieuses de l'enfance. Les distributions de prix en ont retiré une confirmation de leur caractère civique - les enfants ne défilaient plus, mais ils chantaient toujours des hymnes patriotiques - ainsi qu'un rattachement à la Révolution, plus facile à assumer que l'origine jésuite.

41 En 1906-1907, on souligne à nouveau, et avec beaucoup de force, ce besoin de fête laïque, dont les remises de prix fournissent l'occasion. On renoue avec les fonctions pédagogiques de la fête révolutionnaire : réunir, éduquer et récompenser. Le " beau jour des prix » devait, particulièrement chez les grands élèves, combattre celui de la communion solennelle et, de fait, il y a entre les deux cérémonies d'indiscutables analogies : la valeur de rite de passage entre l'enfance et l'âge adulte, la remise d'un livre « viatique », missel ou livre de lecture utile, prolongements festifs familiaux. La génération contemporaine de la fin des distributions de prix, la mienne, a conservé d'elles une double image.Placées sous la seule initiative des communes, elles ont décliné. Progressivement, elles se sont vidées de leur utilité : l'école publique n'a plus craint autant la menace de l'école confessionnelle tandis que la République s'affermissait, l'école unique s'est réalisée, la scolarité s'est allongée, mais pour beaucoup le passage à la vie active s'est effectué sous le signe de l'échec scolaire. Par ailleurs le livre a perdu à la fois son caractère de rareté et son monopole comme 
support d'acquisition de connaissances. Elles ont fini par disparaître à des époques diverses selon les endroits.

Mais une autre image est attachée aux prix, véhiculée par les récits de nos grandsparents, celle des gros volumes rouges à lettres d'or, objets de respect et de fierté. C'est ce souvenir d'un âge un peu mythique qui se présente lorsqu'on parle aujourd'hui de distribution des prix :

"Le rétablissement des distributions de prix serait bénéfique à la fois pour la qualité des études et pour le livre. Ce dernier remonterait dans l'échelle des valeurs. L'enfant prendrait l'habitude de posséder des livres, se constituerait un début de bibliothèque. Pour les rejetons de famille modeste, c'est d'ailleurs le seul moyen d'en avoir. M. Pierre Marchand, le dynamique directeur de Gallimard-Jeunesse, fils d'un ouvrier agricole, a appris à lire dans les livres de prix de ses frères et sceurs. [...] Pas question, hélas, d'en revenir aux beaux volumes reliés en rouge, avec dorures au fer chaud. [...]. Les communes, d'ailleurs, ne tarderaient pas à suivre le mouvement, car les distributions de prix des écoles primaires pourraient être présidées, comme autrefois, par les maires ${ }^{31}$.

\section{NOTES}

1. Tarn, chef-lieu de l'arrondissement, 6544 habitants, au centre d'une région agricole catholique dans laquelle le poids des notables et du clergé est fort.

2. Tarn, arrondissement de Gaillac, 2525 habitants. Ce chef-lieu de canton se trouve dans le vignoble de Gaillac, dans une partie du Tarn où la pratique religieuse est faible.

3. Aristide TORNIER est conseiller général républicain depuis 1870 .

4. De DAINVILlE F., La Naissance de l'humanisme moderne. Paris, Éd. Beauchesne, 1949, p. 148.

5. SNYDERS G., La pédagogie en France aux XVII et XVIII' siècles, Paris, PUF, 1965, p. 49.

6. Journal de Lavaur, 21 août 1881.

7. La deuxième " guerre des manuels », en 1909-1910, a concerné les manuels d'histoire, selon un scénario tout à fait semblable.

8. Il s'agissait des Éléments d'instruction civique et de morale, publiés en 1880 et mis à l'index en en 1882.

9. Les développements de ce conflit à propos des manuels de morale dans le Tarn ont été étudiés par Jean FAURY dans sa thèse: Cléricalisme et anticléricalisme dans le Tarn (1848-1900), Toulouse, Service des publications de l'Université Toulouse Le Mirail, 1980, pp. 123-162.

10. On trouve dans la série $T$ des Archives départementales des listes par arrondissement de propositions commentées pour les présidences des prix adressées au préfet par les inspecteurs primaires.

11. Cf. REBÉRIOUX M., «1885, la première campagne électorale de Jaurès 》, in Bulletin de la société d'études jaurésiennes, 1961, $\mathrm{N}^{\circ}$ 2, pp. 10-13.

12. Cf. ISAMBERT-JAMATI V., Crises de la société, crises de l'enseignement. Sociologie de l'enseignement secondaire français, Paris, P.U.F., 1970, 400 p., qui étudie à travers les discours des prix les fins poursuivies par les Lycées de 1860 à 1965.

13. Gabriel COMPAYRÉ est député du Tarn depuis 1881. Élu conseiller général de Lavaur depuis 1883, il est vice-président et membre de la commission des finances de l'assemblée départementale dont la majorité vient de passer au parti républicain. 
14. Le Nouvelliste du 17 août 1884 avait, par anticipation, ironisé sur le discours de Gabriel COMPAYRÉ : «Là, devant une assemblée d'enfants, de cuisinières et de républicains béats, son éloquence de charlatan se trouve à l'aise. »

15. Les réponses des préfets à deux enquêtes sur les sociétés et œuvres ayant pour objet de propager l'instruction élémentaire fournissent beaucoup d'informations sur les activités de la Ligue sous l'Ordre moral. Archives nationales, F17 12524, 12525, 12527.

16. Sur cette évolution de la Ligue, voir Christine MORA, «La diffusion de la culture dans la jeunesse des classes populaires en France depuis un siècle, l'action de la Ligue de l'Enseignement ", in Niveaux de culture et groupes sociaux, Paris \& La Haye, Éd. Mouton, 1971, pp. 247-267.

17. Archives nationales, F17 12306-12316 : Prix d'honneur dans les écoles primaires : concessions de volumes (1870-1898).

18. PROST A., L'enseignement en France, 1800-1967, Paris, A. Colin, 1968, p. 280.

19. Alcide PICARD [administrateur de la Caisse des écoles du $5^{\mathrm{e}}$ arrondissement]. Discours prononcé lors de la distribution des prix aux élèves des écoles communales de la rue de Pontoise, Paris, 1887.

20. OZOUF J., Nous les maîtres d'écoles..., Paris, Julliard, 1967, pp. 189-190.

21. ZOLA É., « Les quatre Évangiles », in Vérité, Paris, Fasquelle, 1957, p. 253.

22. L'analyse d'un corpus d'une centaine de titres offerts par le ministère de l'instruction publique entre 1880 et 1900 , de leurs auteurs et de leurs éditeurs, constitue la première partie de notre thèse d'École des chartes: PRÉVOST N., Livres de prix et distributions de prix dans l'enseignement primaire (1870-1914). 1979.

23. KERGOMARD P., L'éducation maternelle à l'école, Paris, Hachette, 1886, t. I, p. 86. - Inspectrice générale des écoles maternelles de 1879 à 1917, elle tenta d'endiguer le gavage intellectuel en usage, de substituer le jeu "travail de l'enfant » à la leçon et de faire reconnaître la spécificité pédagogique des écoles du premier âge.

24. JANET P., Cours de psychologie et de morale $2^{e}$ année: morale théorique et morale pratique, Paris, Delagrave, 1891, p. 132. - C'est un manuel en usage dans les écoles normales primaires.

25. DUFRENNE A., "Cours de pédagogie : de la discipline », in Revue de l'enseignement primaire et primaire supérieur, 1906, 9 avril, p. 249-251. - D'inspiration socialiste, cette revue se définit comme "exactement et exclusivement scientifique, laïque, démocratique, humanitaire et sociale ».

26. BUISSON F., La réforme des distributions de prix, Manuel général de l'instruction publique, 1906, 7 juillet, pp. 459-460.

27. DUFRENNE A., "Cours de pédagogie : de la discipline », in Revue de l'enseignement primaire et primaire supérieur, 1906, 9 avril, p. 150.

28. Ces groupes s'expriment dans la Revue de l'enseignement primaire et primaire supérieur, dans Le Volume (mouvement amicaliste) et l'Émancipation de l'instituteur (organe du mouvement syndical).

29. GERMOUTY H., Les distributions de prix, Le Volume, 1907, 29 juin, p. 224.

30. ibid, p. 218.

31. CAHART P., Le livre français a-t-il un avenir? Rapport au ministre de la Culture et de la Communication, Paris, Documentations françaises, 1987, p. 31. 


\section{RÉSUMÉS}

Non disponible

Not available

INDEX

Keywords : Languedoc, primary school, scholastic awards ceremony, Tarn, third republic Mots-clés : distribution des prix, école primaire, IIIe république, Languedoc, Tarn

AUTEUR

NICOLE LE-POTTIER-PRÉVOST

Université de Toulouse Le Mirail 